BMJ Open

Diabetes

Research

\& Care

\title{
Metabolic and energetic benefits of microRNA-22 inhibition
}

\author{
Marc Thibonnier (D) , ${ }^{1}$ Christine Esau, ${ }^{1}$ Sujoy Ghosh, ${ }^{2}$ Edward Wargent, ${ }^{3}$ \\ Claire Stocker ${ }^{3}$
}

To cite: Thibonnier M, Esau C, Ghosh S, et al. Metabolic and energetic benefits of microRNA-22 inhibition. BMJ Open Diab Res Care 2020;8:e001478. doi:10.1136/ bmjdrc-2020-001478

- Supplemental material is published online only. To view please visit the journal online (http://dx.doi.org/10.1136/ bmjdrc-2020-001478).

Received 21 April 2020 Revised 2 July 2020 Accepted 13 August 2020

\section{Check for updates}

C) Author(s) (or their employer(s)) 2020. Re-use permitted under CC BY-NC. No commercial re-use. See rights and permissions. Published by BMJ.

${ }^{1}$ R\&D, AptamiR Therapeutics, Inc, Naples, Florida, USA ${ }^{2}$ Centre for Computational Biology and Program in Cardiovascular \& Metabolic Disorders, Duke-NUS Medical School, Singapore

${ }^{3}$ Clore Laboratory, University of Buckingham, Buckingham, UK

Correspondence to Dr Marc Thibonnier; mthibonnier@aptamir.com

\section{ABSTRACT}

Introduction We previously demonstrated in primary cultures of human subcutaneous adipocytes and in a mouse model of diet-induced obesity that specific microRNA-22-3p antagomirs produce a significant reduction of fat mass and an improvement of several metabolic parameters. These effects are related to the activation of target genes such as KDM3A, KDM6B, PPARA, PPARGC1B and SIRT1 involved in lipid catabolism, thermogenesis, insulin sensitivity and glucose homeostasis.

Research design and methods We now report a dedicated study exploring over the course of 3 months the metabolic and energetic effects of subcutaneous administration of our first miR-22-3p antagomir drug candidate (APT-110) in adult C57BL/6 male mice. Body composition, various blood parameters and energy expenditure were measured at several timepoints between week 12 and week 27 of age.

Results Weekly subcutaneous injections of APT-110 for 12 weeks produced a sustained increase of energy expenditure as early as day 11 of treatment, a significant fat mass reduction, but no change of appetite nor physical activity. Insulin sensitivity as well as circulating glucose, cholesterol and leptin were improved. There was a dramatic reduction of liver steatosis after 3 months of active treatment. RNA sequencing revealed an activation of lipid metabolism pathways in a tissue-specific manner. Conclusions These original findings suggest that microRNA-22-3p inhibition could lead to a potent treatment of fat accumulation, insulin resistance, and related complex metabolic disorders such as obesity, type 2 diabetes mellitus and non-alcoholic fatty liver disease.

\section{INTRODUCTION}

Type 2 diabetes and related chronic diseases resulting from human obesity are major and global public health and financial problems due to sedentary lifestyle and excessive consumption of energy-dense foods rich in saturated lipids and carbohydrates. Current medical treatments of obesity have poor benefit-to-risk profiles, fail to reach their long-term goals and do not meet patients' expectation. ${ }^{1}$ Several medications are usually required to address the various defects contributing to hyperglycemia, and adherence to diabetes multiple therapies is difficult to sustain. ${ }^{2}$ Furthermore, there is currently

\section{Significance of this study}

What is already known about this subject?

- Current therapies aiming at reducing calorie intake and/or absorption to treat obesity and related cardiometabolic disorders have poor efficacy/safety profiles.

- Strategies to increase calorie expenditure and lipid oxidation are currently actively pursued.

What are the new findings?

- A microRNA antagomir, with a one drug-several targets mechanism of action, achieved in the mouse model of diet-induced obesity a significant reduction of fat mass while increasing energy expenditure.

- Additional benefits include improved insulin sensitivity and reduced liver steatosis.

\section{How might these results change the focus of} research or clinical practice?

- Oligonucleotide therapeutics are a promising new class of drugs for the treatment of complex diseases such as diabesity and non-alcoholic fatty liver disease/non-alcoholic steatohepatitis in relation to their mechanism of action.

- These findings need to be confirmed in clinical trials.

no drug approved for the treatment of nonalcoholic fatty liver disease (NAFLD). Therefore, there is a need for a convenient, effective and safe medical treatment of fat accumulation and related metabolic disorders that should significantly reduce healthcare costs and improve and save human lives.

The recent rediscovery of brown adipose tissue (BAT) in adult humans, and the ability to generate brown-like adipocytes ('beige' or 'brite') from white adipose tissue (WAT) depots, has renewed interest in this area of biology. ${ }^{3}$ Promoting 'browning' of subcutaneous WAT is a promising strategy for treating human fat accumulation. ${ }^{4}$ Pharmacological activation of BAT/beige fat may hold promise for combating metabolic diseases such as obesity, diabetes mellitus, dyslipidemia and NAFLD. ${ }^{5}$

MicroRNAs (miRNAs) play important roles in the pathogenesis of many diseases 
including cancers, atherosclerosis, cardiovascular, and metabolic disorders. ${ }^{6}$ Circulating miRNAs have been identified as markers of NAFLD/non-alcoholic steatohepatitis (NASH), liver fibrosis and cancer. ${ }^{7-9}$ miRNA analogs are attractive drug candidates because the simultaneous modulation of many target genes by a single miRNA analog may provide effective therapies for complex diseases such as diabetes, obesity and NAFLD. ${ }^{10}$ For instance, several miRNAs have been implicated in the regulation of insulin signaling, ${ }^{11}$ mitochondrial functions and obesity. ${ }^{12}$

In both mouse models and human subjects, the activation of beige adipocytes is associated with several metabolic benefits, such as reduced obesity, diminished lipotoxicity and increased insulin sensitivity. ${ }^{13}$

Our previous work in silico, in vitro and in vivo identified miR-22-3p as a good metabolic target. ${ }^{14}$ In silico screening revealed several conserved mRNA targets for miR-22-3p, including KDM3A, KDM6B, PPARA, PPARGGC1, PRDM16 and SIRT1. In primary cultures of human subcutaneous adipocytes, inhibition of miRNA22-3p by several complementary antagomirs resulted in increased lipid oxidation, mitochondrial activity, and energy expenditure (EE). Significant increases of mitochondrial mass/activity and UCP1 immunostaining were noted in the presence of an miR-22-3p antagomir. According to the Kyoto Encyclopedia of Genes and Genomes (KEGG) resource, the Peroxisome proliferatoractivated receptor (PPAR) signaling pathway is activated in human adipocytes in culture in the presence of an miR-22-3p antagomir. Subsequently, we conducted several studies to explore the metabolic effects of miR-22 inhibition in diet-induced obesity (DIO) C57BL/6J male mice of various ages. Reduction of weight gain on a high-fat diet was observed in young adult mice treated for 8 weeks with various miR-22 antagomirs. Prevention of obesity induced by high-fat diet was noted in mature adult mice treated for 8 weeks with miR-22 antagomirs.

\section{RESEARCH DESIGN AND METHODS}

A dedicated study was conducted in adult C57BL/6 male mice over the course of 3 months at the University of Buckingham Institute of Translational Medicine to assess the energetic and metabolic responses to a treatment with the custom-made APT-110 miR-22-3p antagomir.

\section{In vivo experiments}

DIO male C57BL/6J mice purchased from the Jackson Laboratory were housed at $24^{\circ} \mathrm{C}-26^{\circ} \mathrm{C}$ with lights on at 08:00 and off at 20:00. They were fed at weaning on chow (Beekay Rat and Mouse Diet No 1), and from 6 weeks of age were started on a 60\% high-fat diet (Research Diet D12492). At the age of 12 weeks, the mice were allocated to treatment groups so that the mean and SD for body weight, glucose and insulin were similar across groups (12 animals per group, 2 animals of the same group per cage). After acclimation for 2 weeks, the mice were administered single subcutaneous injections of saline or the APT-110 miR-22-3p antagomir $(15 \mathrm{mg} / \mathrm{kg})$ in the left inguinal fat pad (injections on days 0,2 , and 4 of week 1 of treatment, then once a week for a total of 12 weeks) while they remained on $60 \%$ high-fat diet.

Body weights were measured weekly. Food consumption per cage was measured daily. Blood samples were collected from the cut tip of the tail after the application of lignocaine gel during the in-life phase of the study. For plasma preparation, blood was collected in EDTA-coated microvettes for measurement of plasma analytes and was stored on ice, before centrifugation at $\sim 5000 \times g$ for $5 \mathrm{~min}$. The resulting plasma was stored at $-80^{\circ} \mathrm{C}$ until required. Multiple freeze/thaw cycles were avoided. Blood glucose concentration was analyzed as previously reported. ${ }^{15}$ Plasma insulin (Cat \#90080; Crystal Chem, Downers Grove, Illinois, USA), leptin (Cat \#90030; Chrystal Chem), adiponectin (Cat \#47-ADPMS-E01, Alpco Diagnostics, Salem, New Hampshire, USA), Non-esterified fatty acids (NEFA) (Cat \#NEFA-HR(2); Wako Diagnostics, Mountain View, California, USA), alanine aminotransferase (ALT) (Cat \#AL1205, Randox, Crumlin, UK) and aspartate transaminase (AST) (Cat \#AS1202, Randox), total cholesterol (Cat \#CH200, Randox) and triglycerides (Cat \#TR210, Randox) were measured as per manufacturers' recommendations.

Oral glucose tolerance test (OGTT) was performed as follows: 6 hours prior to the start of the glucose tolerance test (09:00), food was removed and animals were given clean cages. Mice were dosed with glucose at $\mathrm{T}=0 \mathrm{~min}$. Glucose was dosed by oral gavage at a dose of $2.5 \mathrm{~g} / \mathrm{kg}$ orally. Blood samples were taken for analysis of glucose concentration at $-30,0,30,60,120$ and 180 min relative to glucose administration. Blood samples were also taken at -30 and +30 min for insulin analysis. Food was returned at the end of the tolerance test.

Mice EE was measured by open-circuit calorimetry with the animals in their home cages. ${ }^{1516}$ Recording of physical activity was done in mice that were kept in their original cages. Recordings were taken using an infrared recorder linked to a laptop. Recordings were made on the hour every hour from 19:00 until 08:00. Each recording lasted $10 \mathrm{~min}$. Analysis of activity was done by virtually drawing two lines across each cage (thus dividing them into three equal parts). Recordings were analyzed by eye, and the number of times a line was broken by a mouse in each 10 min segment was scored.

Body fat and lean content were measured using a Minispec LF90II nuclear magnetic resonance (Bruker Corporation, Fremont, California, USA). The mice were gently restrained, sufficient to keep them quiescent during this non-invasive technique.

At the end of the study, liver, heart, inguinal, perirenal, epididymal and subscapular fat samples were collected, weighed, and then frozen for future gene expression analysis; or placed in 10\% neutral buffered formalin solution, then washed in Phosphate-buffered saline (PBS) at $\mathrm{pH} 7.4$ and transferred to $70 \%$ ethanol for 
subsequent processing for histological analyses. Spleens were weighed and discarded. Blood was collected and processed into serum or plasma aliquots at the time of necropsy by cardiocentesis.

\section{miR-22 antagomir}

The miR-22-3p antagomir used in this study was designed by AptamiR Therapeutics and custom-synthesized (US Patent 62/329,537 on 'Inhibition of mir-22 miRNA by APT-110' initially published on 2 November 2017, WO2017/187426 A1). It is an 18-mer single-stranded oligonucleotide complementary to nucleotides 2-19 of miR-22-3p with phosphorothioate, locked nucleic acid, 2'0-methyl, DNA and 5-methyl-cytosine modifications. For subcutaneous dosing in the left inguinal area, APT-110 $(15 \mathrm{mg} / \mathrm{kg})$ was prepared in saline $(0.9 \% \mathrm{NaCl})$. Control animals received the saline vehicle alone $(5 \mathrm{~mL} / \mathrm{kg})$.

\section{Imaging analyses}

Measurement of cells' perimeter and area used the Image J image processing program developed by the National Institutes of Health (NIH) (https://imagej.nih.gov/).

\section{RNA sequencing analyses}

RNA sequencing was performed at the Genome Sequencing and Analysis Facility at the University of Texas at Austin on an Illumina HiSeq 4000 system following the manufacturer's protocol. RNA data analyses were performed by SG at Duke-NUS Medical School, Singapore.

Differentially expressed genes from fat (nominal $\mathrm{p}<0.01$ ) and liver (nominal $\mathrm{p}<0.001$ ) gene expression data were subjected to pathway over-representation analysis via the Enrichr tool ${ }^{17}$ (http://amp.pharm.mssm. edu/Enrichr/). A total of 1257 and 1495 genes were analyzed from fat and liver, respectively. Pathway enrichment was further investigated via the KEGG pathway database $^{18} \quad$ (www.genome.jp/kegg/pathway.html) and the WikiPathways database ${ }^{19}$ (wikipathways.org). Pathways with a false discovery rate $<0.1$ were considered to be significantly enriched for differentially expressed genes. A subset of the significantly enriched KEGG pathways (adjusted $\mathrm{p}<1.5 \mathrm{E}-05$ ) were further visualized via meanaverage (MA) plots.

\section{Statistical analyses}

Results given in the text and data points in the figures are shown as mean \pm SEM. Statistical analysis used analysis of variance and Student's t-test, unless non-parametric tests were selected, based on data distribution (GraphPad Prism V.8.4). No animal data were excluded from the study protocol and data analyses.

\section{RESULTS}

APT-110 reduces body fat mass and total body weight but not food consumption

Administration of the APT-110 miR-22-3p antagomir reduced body weight gain in mice on $60 \%$ high-fat diet when compared with the vehicle-treated animals.

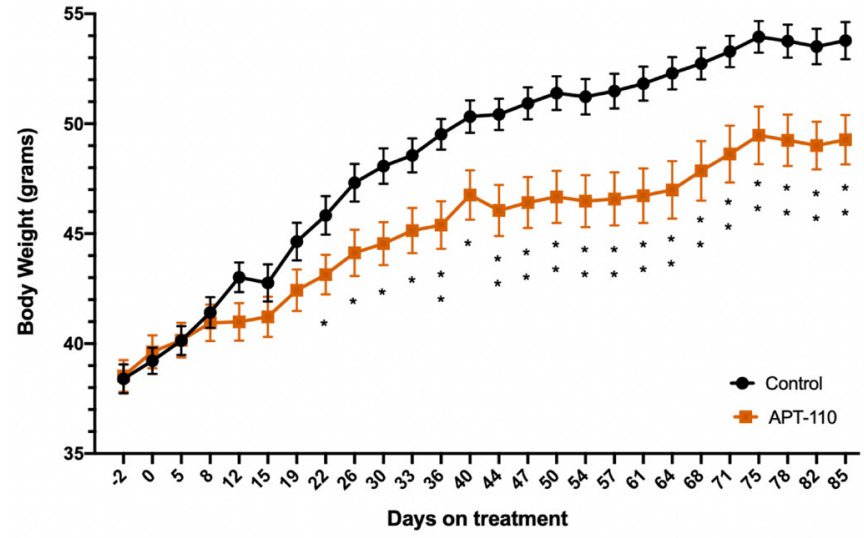

Figure 1 Body weight profile of adult mice on $60 \%$ high-fat diet alone (saline, black line and circles) or in the presence of the APT-110 miR-22-3p antagomir (brown line and squares) during 12 weeks of treatment, starting at week 15 of age $\left({ }^{*} p<0.05 ;{ }^{* *} p<0.01\right)$.

The body weight difference became statistically significant as of the third week on treatment. At the end of 12 weeks on high-fat diet (27-week-old mice), the mice treated with APT-110 gained significantly less weight $(-30 \%, \mathrm{p}=0.0041)$ than the mice receiving saline injections (figure 1). Body composition analysis by Nuclear Magnetic Resonance (NMR) at the end of the study (day 82 of treatment) showed that the body weight difference was due to a fat mass difference only $(-12 \%$ in the APT-110 group, $\mathrm{p} \leq 0.001)$, whereas lean mass was identical in both groups (online supplemental figure 1). The difference in body weight and fat mass in the APT-110 dosed group was statistically the same. There was no difference in food consumption between the two groups that could have explained the body weight differences (total consumption of $229 \pm 6 \mathrm{~g}$ per cage vs $223 \pm 5$ g per cage, APT-110 vs saline groups, respectively).

\section{APT-110 increases EE but not physical activity}

The first 24-hour EE recording performed on day 10 of treatment did not show a difference between the saline and APT-110 groups (figure 2A). However, an increase in EE was observed in the APT-110 group as early as day 11 (figure 2B) and was confirmed on days 28, 29 and 52 (figure 2C) of active treatment. The total daily EE measured from day 11 to day 52 was on average $13 \%$ greater on APT-110 treatment (figure 2C). Furthermore, after intraperitoneal injection of a maximal dose $(0.5 \mathrm{mg} / \mathrm{kg})$ of the $\beta_{3}$-adrenoceptor agonist CL316243 on day 53, EE increased both in the control and the APT-110 groups $(+27.25 \pm 2.34 \mathrm{vs}+29.03 \pm 5.97 \mathrm{kj} / \mathrm{animal} /$ day), but it remained greater in the APT-110 group than in the control group $(\mathrm{p}=0.0019)$, suggesting that miR-22-3p inhibition had increased the total amount of thermogenic adipose tissue that is still responsive to $\beta_{3}$-adrenoceptor agonist stimulation (figure 2D). 
A
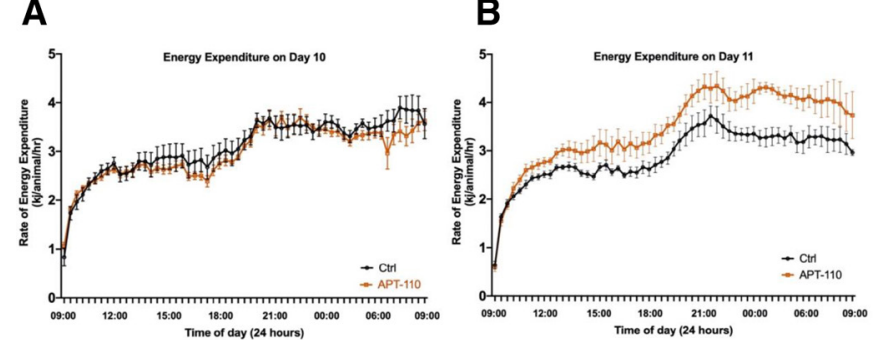

C
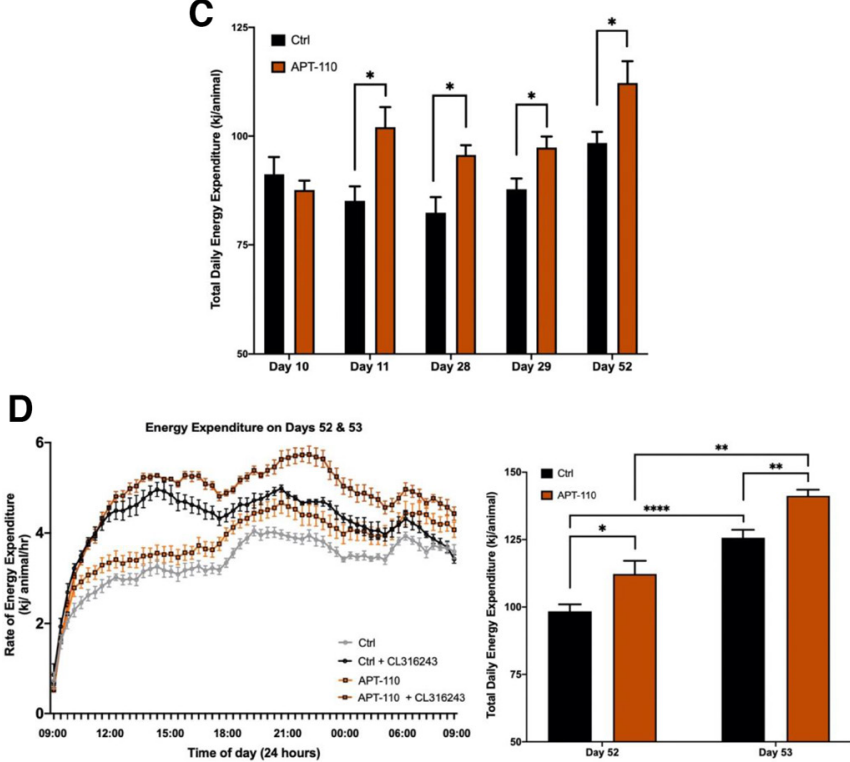

Figure 2 Energy expenditure measured over 24 hours at different timepoints of the study in mice receiving saline injections (black lines) or the APT-110 miR-22-3p antagomir (brown lines). (A) Total energy expenditure measured on day 10. (B) Total energy expenditure measured on day 11. (C) Total energy expenditure measured over 24 hours on different days in mice receiving saline injections (black columns) or the APT-110 miR-22-3p antagomir (brown columns). (D) Total energy expenditure measured over 24 hours on days 52 and 53 in mice receiving saline injections (gray and black columns) or the APT-110 miR-22-3p antagomir (brown and red columns). Measurements on day 53 were made in the presence of a $0.5 \mathrm{mg} / \mathrm{kg}$ intraperitoneal dose of the beta-3 receptor agonist CL316243. Ctrl, control $\left({ }^{\star} \mathrm{p}<0.05 ;{ }^{* \star} \mathrm{p}=0.01\right.$; **** $\mathrm{p} \leq 0.0001)$.

There was no difference in night-time physical activity between the two groups that could have explained the EE and body weight differences $(57 \pm 8$ line breaks per cage vs $54 \pm 8$ line breaks per cage, APT-110 vs saline groups, respectively).

\section{APT-110 improves glucose homeostasis and insulin sensitivity}

Blood glucose, plasma insulin, leptin and adiponectin were measured after fasting the mice for 6 hours on days $-2,44$ and 79 of treatment. Blood glucose, plasma insulin and leptin levels were significantly lower on APT-110 treatment, whereas plasma adiponectin levels were similar across both groups (table 1).

Calculation of the fasting insulin/glucose at different timepoints during the course of the study illustrates the development of insulin resistance over time in the control group on $60 \%$ fat diet, whereas the group treated with the APT-110 mir-22-3p antagomir maintains the same level of insulin sensitivity (online supplemental figure 2).

OGTTs were performed during week 5 (day 40) and week 12 (day 75) of treatment. The APT-110 miR-22-3p antagomir significantly improved glucose tolerance at week 5 (blood glucose total area under the curve (AUC): $92.78 \pm 5.75$ vs $76.83 \pm 2.80$, control vs APT- $110 ; \mathrm{p}=0.0139$ ) and even more at week 12 (blood glucose total AUC: 79.44 \pm 4.26 vs 48.64 \pm 3.18 , control vs APT-110; $\mathrm{p}<0.0001$ ) (figure 3A,B). Fasting insulin levels measured at OGTT timepoint -30 were dramatically lower in the APT-110 group than in the saline group after both 5 and 12 weeks of treatment as were the glucose-stimulated plasma insulin concentrations measured at OGTT timepoint +30 .

\section{APT-110 effects on lipid profile}

Circulating lipid profile was measured on day 85 at the end of the last week of treatment after a 6-hour fast (table 2). The group on the APT-110 miR-22-3p antagomir displayed reduced cholesterol and increased NEFA levels. These changes reflect the increased metabolic rate in the APT-110treated mice, an increased lipolytic rate leading to the increase in the release of fatty acids into the blood, thus increasing beta oxidation. Liver function tests were similar and within the normal range in both groups (table 2).

\section{APT-110 effects on liver weight and appearance}

Assessment of organ weights revealed a significant reduction of liver size (both absolute or per cent of body weight; $-20 \%, 2.442 \pm 0.127$ vs $3.057 \pm 0.114 \mathrm{~g}$; $\mathrm{p}<0.0001$ ) in the APT-110 group, whereas the sizes of the heart and

\begin{tabular}{|c|c|c|c|c|c|}
\hline Day of treatment & Group & $\begin{array}{l}\text { Blood glucose } \\
\text { (mmol/L) }\end{array}$ & $\begin{array}{l}\text { Plasma insulin } \\
\text { (pmol/L) }\end{array}$ & $\begin{array}{l}\text { Plasma leptin } \\
\text { (nmol/L) }\end{array}$ & $\begin{array}{l}\text { Plasma adiponectin } \\
\text { (ng/mL) }\end{array}$ \\
\hline \multirow[t]{2}{*}{ Day -2} & Control & $6.65 \pm 0.21$ & $172 \pm 21$ & $3.27 \pm 0.31$ & $3.20 \pm 0.15$ \\
\hline & APT-110 & $6.69 \pm 0.27$ & $179 \pm 18$ & $3.11 \pm 0.45$ & $3.16 \pm 0.13$ \\
\hline \multirow[t]{2}{*}{ Day 44} & Control & $10.94 \pm 0.34$ & $837 \pm 91$ & $8.23 \pm 0.62$ & $3.04 \pm 0.13$ \\
\hline & APT-110 & $9.01 \pm 0.19^{*}$ & $295 \pm 25^{*}$ & $4.85 \pm 0.42^{*}$ & $2.66 \pm 0.15$ \\
\hline \multirow[t]{2}{*}{ Day 79} & Control & $7.31 \pm 0.20$ & $843 \pm 47$ & $7.81 \pm 1.09$ & $3.82 \pm 0.24$ \\
\hline & APT-110 & $5.71 \pm 0.18^{*}$ & $220 \pm 17^{*}$ & $4.21 \pm 0.54^{*}$ & $3.16 \pm 0.13$ \\
\hline
\end{tabular}

${ }^{*} \leq 0.0001$ vs control group at the same timepoint. 
A

OGTT and insulin levels during week 5
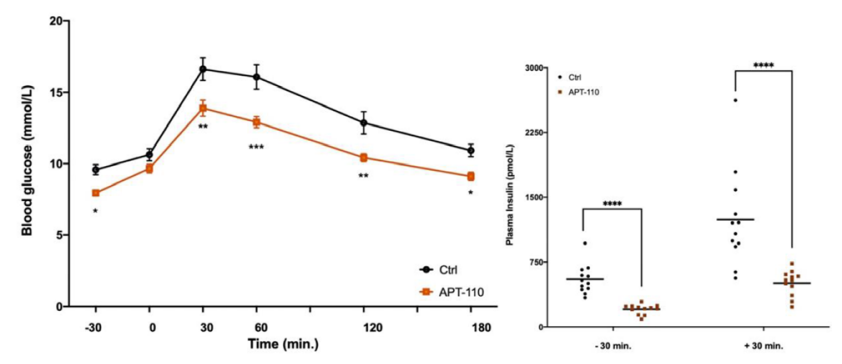

B

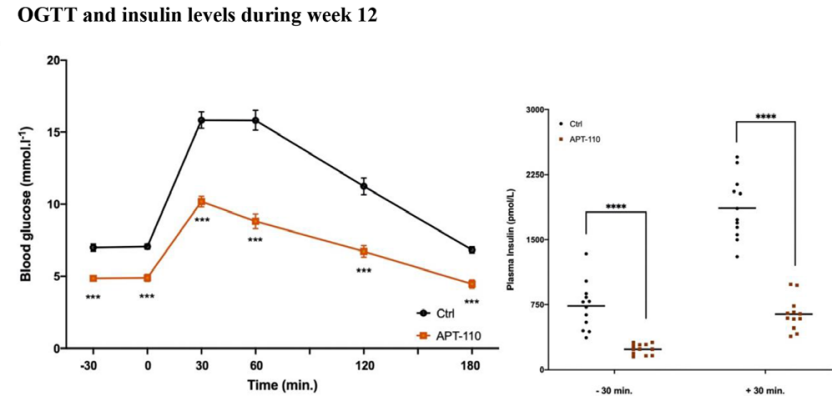

Figure 3 Oral glucose tolerance test (OGTT) and insulin levels during week 5 and week 12 of treatment in mice receiving saline injections (black line) or the APT-110 miR-22$3 p$ inhibitor (brown line). (A) OGTT and insulin levels during week 5. (B) OGTT and insulin during week 12. ${ }^{*} p \leq 0.05$; ${ }^{* *} \mathrm{p} \leq 0.01 ;{ }^{* * *} \mathrm{p} \leq 0.001 ;{ }^{* * *} \mathrm{p} \leq 0.0001$. Ctrl, control.

the spleen were similar in the saline and APT-110 groups. Treatment for 12 weeks with the APT-110 miR-22-3p antagomir produced a marked reduction in fatty infiltration of the liver in these mice on a high-fat diet (figure 4). The average fat content was calculated at $27.34 \%$ in the saline group and $18.57 \%$ in the APT- 110 group $(\mathrm{p} \leq 0.0001)$.

\section{APT-110 effects on gene expression}

RNAseq analyses of organs and tissues after 3 months of treatment with the APT-110 miR-22-3p antagomir showed the following changes versus the saline control group. The volcano plots show significant differences between the two conditions that are tissue-specific/organ-specific (online supplemental figure 3A). For instance, differentially expressed genes in the liver (adjusted $\mathrm{p}<0.01$ ) were subjected to KEGG pathway enrichment analysis via the Enrichr tool (online supplemental figure 3B). A subset of the significantly enriched KEGG pathways (adjusted $\mathrm{p}<1.5 \mathrm{E}-05$ ) were visualized via MA plots. Pathway names are indicated on the top of each plot. In each plot, pathway genes are shown as filled orange circles. All

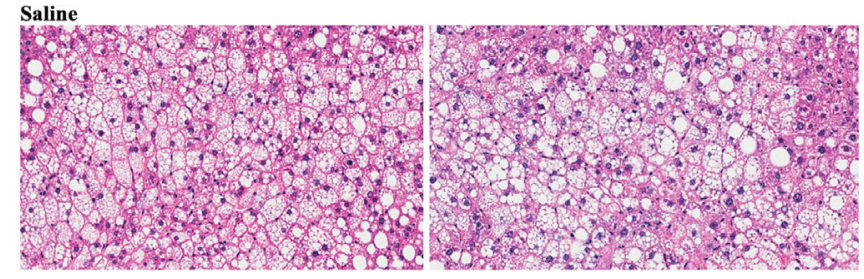

APT-110

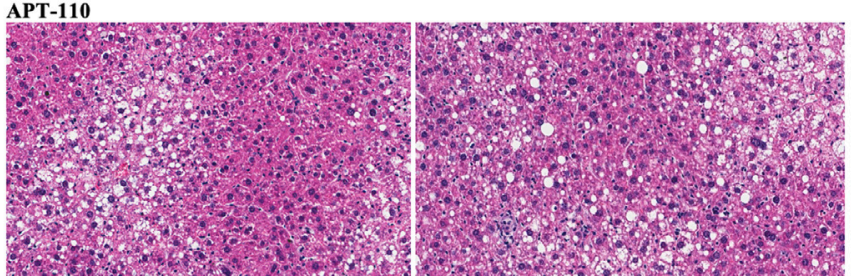

Figure 4 Histological appearance of the liver (H\&E staining) at the end of 12 weeks of treatment in mice receiving saline injections or the APT-110 miR-22-3p inhibitor (two samples from each group are shown).

other non-pathway genes from the RNAseq study are shown as tan, open circles (background). Comparison of the plots between the liver and the inguinal samples shows a general downregulation of fatty acid biosynthetic pathway genes in the APT-110-treated livers and a general upregulation of fatty acid metabolism genes in the APT-110-treated inguinal fat (log2FC is comparing APT-110 with controls) (online supplemental figure 3C,D). Of note, the complement and coagulation pathways were downregulated in the APT-110-treated subscapular and inguinal samples.

\section{DISCUSSION}

The concept of modulation of energy-expending adipocytes in human adults is now firmly established. ${ }^{20}$ Increase of $\mathrm{EE}$ as an effective treatment of obesity and related disorders is appealing as a target for drug research and development. ${ }^{21} \mathrm{~A} 10 \%-15 \%$ increase of EE is believed to be sufficient to achieve significant weight and fat mass reduction while providing meaningful improvement of several metabolic parameters. ${ }^{22} 23$

miRNAs are involved in many metabolic functions, including adipogenesis, obesity, browning of adipocytes, insulin resistance, and lipid and glucose metabolism, and they have been shown to be dysregulated in various cardiometabolic diseases. ${ }^{24-30}$

miR-22-3p is a unique, conserved and universal miRNA that is highly expressed in human and murine

Table 2 Circulating lipid profile and liver enzymes at the end of the metabolic/energy expenditure study

\begin{tabular}{|c|c|c|c|c|c|c|}
\hline Day of treatment & Group & $\begin{array}{l}\text { Cholesterol } \\
\text { (mmol/L) }\end{array}$ & $\begin{array}{l}\text { NEFA } \\
\text { (mmol/L) }\end{array}$ & $\begin{array}{l}\text { Triglycerides } \\
\text { (mmol/L) }\end{array}$ & $\begin{array}{l}\text { ALT } \\
\text { (U/L) }\end{array}$ & $\begin{array}{l}\text { AST } \\
\text { (U/L) }\end{array}$ \\
\hline Day 85 & Control & $5.93 \pm 0.14$ & $1.38 \pm 0.06$ & $1.28 \pm 0.08$ & $57 \pm 5$ & $57 \pm 6$ \\
\hline $\mathrm{P}$ value & & $<0.0001$ & 0.003 & 0.11 & n.s. & n.s. \\
\hline
\end{tabular}

ALT, alanine aminotransferase; AST, aspartate transaminase; NEFA, non-esterified fatty acids; n.s., not significant. 
subcutaneous adipocytes. ${ }^{31}$ Furthermore, miR-22-3p is transported by adipocyte exosomes. ${ }^{32}{ }^{33}$ Castaño et $a e^{34}$ have recently shown that DIO in mice modifies the miRNA profile of plasma exosomes, including increases in miR-122, miR-192, miR-22-3p, miR-27a-3p and miR27b-3p, which modulate glucose and lipid metabolism. Their findings highlight the central role of obesityassociated exosomal miRNAs in the etiopathology of insulin resistance, glucose intolerance and dyslipidemia. Recent elegant studies have shown that adipose tissue constitutes a major source of circulating exosomal miRNAs regulating gene expression in distant tissues like liver and muscle where they modulate insulin sensitivity and inflammation, acting as a novel type of adipokines. ${ }^{3536}$

A role for miR-22 in lipid metabolism, EE and fat mass has been illustrated by several independent academic groups. ${ }^{97-41}$ Our findings suggest that miR-22-3p antagonism produces in mice several metabolic and energetic benefits (fat mass reduction, moderate increase of EE, improvement of insulin sensitivity and glucose homeostasis, reduction of circulating cholesterol and leptin despite the ad libitum consumption of a $60 \%$ high-fat diet, and no change of physical activity). These effects are linked to the antagonism of miR-22-3p not only in the organs and tissues where it is expressed such as the adipose tissue (direct effects), but also in distant organs and tissues such as the liver, where miR-22-3p is delivered via circulating exosomes (paracrine effects). Hu et al recently reported that human and mouse fatty livers have elevated miR-22 levels and reduced target genes such as PPARA, PGC1A and also FGFR $1 .^{42}$ In their hands, miR-22 delivery increased steatosis, whereas miR-22 antagomir was able to reduce alcoholic steatosis and Western dietinduced steatosis and insulin resistance in mice. Based on other investigators' publications and our own work, a model of miR-22-3p target genes involved in lipid oxidation, mitochondrial functions, thermogenesis, glucose metabolism, adipocyte differentiation, inflammation and antioxidation is proposed in online supplemental figure 4. This set of 25 target genes was analyzed with the metaMIR in silico tool, a combinatorial approach using machine learning-based meta-analysis of established algorithms. ${ }^{43}$ This independent analysis revealed that miR-22-3p was the miRNA that achieved the highest final score for a positive combination of 14 target genes (online supplemental table 1).

In addition to a significant reduction of circulating cholesterol in the miR-22-3p antagomir-treated group, it is worth noting the increase of circulating NEFA. The balance of WAT lipolysis and lipogenesis is the prime energy reserve, ${ }^{44}$ and as it is NEFA that are uptaken into tissues for energy provision it is the mobilization of NEFA that represents the metabolic adaptation to increased energy requirement. ${ }^{45}$ Obesity causes imbalance in lipogenesis/lipolysis resulting in elevated plasma NEFA, which has been shown to be an important factor in the development of insulin resistance. ${ }^{46}$ In this experiment, while the treated group had elevated plasma NEFA, they also had improved insulin sensitivity along with increased EE. Thus, the increased availability of NEFA in the presence of the miR-22-3p antagonism most likely reflects an increased demand for energy provision due to elevated levels of lipid metabolism in the inguinal WAT and possibly an improvement in the lipogenesis/lipolysis balance, although further studies would be required to address this sufficiently.

The effect of the miR-22-3p antagomir on liver steatosis in these animals on a high-fat diet is notable. Potential effects on prevention and regression of steatohepatitis and liver fibrosis will be assessed in animals of various ages placed on a high-fat/high-sucrose diet or a 'Western' diet.

As expected, the administration of a specific miR-22-3p antagomir produces expression changes of several genes (one drug-multiple targets concept) that should facilitate the treatment of complex diseases. ${ }^{47}$ Our gene profiling data confirm that a specific miR-22-3p antagomir produces change of several metabolic pathways in a tissue-specific manner. For instance, the simultaneous downregulation of fatty acid biosynthetic pathway genes in the liver and the upregulation of fatty acid metabolism genes in the inguinal fat in the presence of APT-110 should have facilitated the reduction of fat accumulation in these organs. These findings call for extensive lipidomic and metabolomic profiling of various tissues in future studies with oligonucleotide therapeutics.

A novel model of cardiometabolic-based chronic disease (CMBCD) including adiposity-based chronic disease (ABCD, defined as abnormalities in the amount, distribution, and function of adipose tissue) and dysglycemiabased chronic disease (DBCD) is now proposed 'to provide a basis for early and sustainable, evidence-based therapeutic targeting to promote cardiometabolic health and mitigate the development and ravages of cardiovascular disease'. ${ }^{48} \mathrm{CMBCD}$ focuses on three primary drivers (genetics, environment, and behavior) and two metabolic drivers (adiposity and dysglycemia). Insulin resistance is at the intersection between $\mathrm{ABCD}$ and $\mathrm{DBCD}$, and further weight gain can worsen insulin resistance and intensify inflammation, oxidative stress, and glucose intolerance. Adiposity leads to pancreatic beta-cell dysfunction and viability via glucotoxicity, lipotoxicity, increased oxidative stress, and inflammation, setting the stage for hyperglycemia and progression to pre-diabetes and type 2 diabetes mellitus. Our study suggests that miRNA-22-3p antagonism has a significant impact on $\mathrm{ABCD}$ and $\mathrm{DBCD}$, especially at the level of insulin resistance.

At a time when triple-drug combinations are approved for the treatment of diabetes and triple incretin/glucagon agonist peptides are being developed for diabesity, ${ }^{49}$ oligonucleotide therapeutics agents such as miR-22-3p antagomirs may play a role as an effective and convenient simplified treatment of diabesity and NAFLD/NASH.

Acknowledgements We would like to thank the following individuals: Dr Huntington F Willard, Director, Geisinger National Precision Health and Howard 
Hughes Medical Institute Professor, for his scientific input and indepth discussions on microRNAs; Dr Daniel Ricquier, Professor at University Paris Descartes and a member of the French Academy of Sciences, for sharing his expertise on thermogenesis and his critical reading of the manuscript; Dr Eric Clauser, Director of the INSERM School, for his indepth review of the data and manuscript; and Dr Jonathan Arch, Emeritus Professor, University of Buckingham, whose skills and knowledge significantly improved the quality of the manuscript.

Contributors MT: conceptualization, validation, formal analyses, writing, visualization, supervision, project administration and funding acquisition. CE: methodology, validation, formal analyses, investigations, data curation, review and editing, and supervision. SG: bioinformatic analyses of NGS data. EW and CS: methodology, validation, formal analyses, investigation, data curation, review and editing, and supervision at the University of Buckingham Institute of Translational Medicine. MT is the guarantor of the work as a whole.

Funding All funding for this R\&D work came from AptamiR Therapeutics.

Competing interests Ownership: MT is the founder and a shareholder of AptamiR Therapeutics. Intellectual property: MT is the inventor of several patents assigned to AptamiR Therapeutics.

\section{Patient consent for publication Not required.}

Ethics approval This animal study was performed according to an IACUC-approved protocol and in compliance with the Guide for the Care and Use of Laboratory Animals (National Research Council, 2011) in OLAW-assured and AAALAC-accredited facilities at the University of Buckingham, UK.

Provenance and peer review Not commissioned; externally peer reviewed.

Data availability statement All data relevant to the study are included in the article or uploaded as supplemental information.

Open access This is an open access article distributed in accordance with the Creative Commons Attribution Non Commercial (CC BY-NC 4.0) license, which permits others to distribute, remix, adapt, build upon this work non-commercially, and license their derivative works on different terms, provided the original work is properly cited, appropriate credit is given, any changes made indicated, and the use is non-commercial. See: http://creativecommons.org/licenses/by-nc/4.0/.

\section{ORCID iD}

Marc Thibonnier http://orcid.org/0000-0002-4546-180X

\section{REFERENCES}

1 Kim DD, Basu A. Estimating the medical care costs of obesity in the United States: systematic review, meta-analysis, and empirical analysis. Value Health 2016;19:602-13.

2 David J, Fonseca V. When should fixed ratio basal insulin/ glucagon-like peptide- 1 receptor agonists combination products be considered? J Diabetes Complications 2019;33:107473.

3 Ikeda K, Maretich P, Kajimura S. The common and distinct features of brown and beige adipocytes. Trends Endocrinol Metab 2018;29:191-200.

$4 \mathrm{Kim} \mathrm{SH}$, Plutzky J. Brown fat and browning for the treatment of obesity and related metabolic disorders. Diabetes Metab $J$ 2016;40:12-21.

5 Rui L. Brown and beige adipose tissues in health and disease. Compr Physiol 2017;7:1281-306.

6 van Rooij E, Kauppinen S. Development of microRNA therapeutics is coming of age. EMBO Mol Med 2014;6:851-64.

7 de Conti A, Ortega JF, Tryndyak V, et al. Microrna deregulation in nonalcoholic steatohepatitis-associated liver carcinogenesis. Oncotarget 2017;8:88517-28.

8 Tessitore A, Cicciarelli G, Del Vecchio F, et al. Microrna expression analysis in high fat diet-induced NAFLD-NASH-HCC progression: study on C57BL/6J mice. BMC Cancer 2016;16:3.

9 López-Riera M, Conde I, Quintas G, et al. Non-Invasive prediction of NAFLD severity: a comprehensive, independent validation of previously postulated serum microRNA biomarkers. Sci Rep 2018;8:10606.

10 Inui M, Martello G, Piccolo S. Microrna control of signal transduction. Nat Rev Mol Cell Biol 2010;11:252-63.

11 Ebrahimi R, Bahiraee A, Niazpour F, et al. The role of microRNAs in the regulation of insulin signaling pathway with respect to metabolic and mitogenic cascades: a review. J Cell Biochem 2019;120:19290-309.

12 Murri M, El Azzouzi H. Micrornas as regulators of mitochondrial dysfunction and obesity. Am J Physiol Heart Circ Physiol 2018;315:H291-302.
13 Paulo E, Wang B. Towards a better understanding of beige adipocyte plasticity. Cells 2019;8. doi:10.3390/cells8121552

14 Thibonnier M, Esau C. Metabolic benefits of microRNA-22 inhibition. Nucleic Acid Ther 2020;30:104-16.

15 Stocker CJ, Wargent E, O'Dowd J, et al. Prevention of dietinduced obesity and impaired glucose tolerance in rats following administration of leptin to their mothers. Am J Physiol Regul Integr Comp Physiol 2007;292:R1810-8.

16 Arch JRS, Trayhurn P. Detection of thermogenesis in rodents in response to anti-obesity drugs and genetic modification. Front Physiol 2013;4:64.

17 Chen EY, Tan CM, Kou Y, et al. Enrichr: interactive and collaborative HTML5 gene list enrichment analysis tool. BMC Bioinformatics 2013;14:128.

18 Kanehisa M. Kegg bioinformatics resource for plant genomics and metabolomics. Methods Mol Biol 2016;1374:55-70.

19 Slenter DN, Kutmon M, Hanspers K, et al. WikiPathways: a multifaceted pathway database bridging metabolomics to other omics research. Nucleic Acids Res 2018;46:D661-7.

20 Celi FS, Le TN, Ni B. Physiology and relevance of human adaptive thermogenesis response. Trends Endocrinol Metab 2015;26:238-47.

21 Lee P, Swarbrick MM, Ho KKY. Brown adipose tissue in adult humans: a metabolic renaissance. Endocr Rev 2013;34:413-38.

22 Cereijo R, Giralt M, Villarroya F. Thermogenic brown and beige/brite adipogenesis in humans. Ann Med 2015;47:169-77.

23 Vosselman MJ, van Marken Lichtenbelt WD, Schrauwen P. Energy dissipation in brown adipose tissue: from mice to men. Mol Cell Endocrinol 2013;379:43-50.

24 Price NL, Fernandez-Hernando C. miRNA regulation of white and brown adipose tissue differentiation and function. Biochim Biophys Acta 1861;2016:2104-10.

25 Xie H, Sun L, Lodish HF. Targeting microRNAs in obesity. Expert Opin Ther Targets 2009;13:1227-38.

26 Arner P, Kulyté A. Microrna regulatory networks in human adipose tissue and obesity. Nat Rev Endocrinol 2015;11:276-88.

27 Abente EJ, Subramanian M, Ramachandran V, et al. MicroRNAs in obesity-associated disorders. Arch Biochem Biophys 2016;589:108-19.

$28 \mathrm{Zhu} \mathrm{H}$, Leung SW. Identification of microRNA biomarkers in type 2 diabetes: a meta-analysis of controlled profiling studies. Diabetologia 2015;58:900-11.

29 Son YH, Ka S, Kim AY, et al. Regulation of adipocyte differentiation via microRNAs. Endocrinol Metab 2014;29:122-35.

30 Hilton C, Neville MJ, Karpe F. MicroRNAs in adipose tissue: their role in adipogenesis and obesity. Int $J$ Obes 2013;37:325-32.

31 Liang Y, Ridzon D, Wong L, et al. Characterization of microRNA expression profiles in normal human tissues. BMC Genomics 2007;8:166.

32 Eguchi A, Lazic M, Armando AM, et al. Circulating adipocyte-derived extracellular vesicles are novel markers of metabolic stress. $J$ Mol Med 2016;94:1241-53.

33 Ying W, Riopel M, Bandyopadhyay G, et al. Adipose Tissue Macrophage-Derived Exosomal miRNAs Can Modulate In Vivo and In Vitro Insulin Sensitivity. Cell 2017;171:e312:372-84.

34 Castaño C, Kalko S, Novials A, et al. Obesity-Associated exosomal miRNAs modulate glucose and lipid metabolism in mice. Proc Natl Acad Sci U S A 2018;115:12158-63.

35 Thomou T, Mori MA, Dreyfuss JM, et al. Adipose-Derived circulating miRNAs regulate gene expression in other tissues. Nature 2017;542:450-5.

36 Lee M-W, Lee M, Oh K-J. Adipose tissue-derived signatures for obesity and type 2 diabetes: adipokines, Batokines and microRNAs. J Clin Med 2019;8:854.

37 Diniz GP, Huang Z-P, Liu J, et al. Loss of microRNA-22 prevents high-fat diet induced dyslipidemia and increases energy expenditure without affecting cardiac hypertrophy. Clin Sci 2017;131:2885-900.

38 Schweisgut J, Schutt C, Wüst S, et al. Sex-Specific, reciprocal regulation of $E R \alpha$ and miR-22 controls muscle lipid metabolism in male mice. Embo J 2017;36:1199-214.

39 Kaur K, Vig S, Srivastava R, et al. Elevated hepatic miR-22-3p expression impairs gluconeogenesis by silencing the Wntresponsive transcription factor Tcf7. Diabetes 2015;64:3659-69.

40 Kaur K, Pandey AK, Srivastava S, et al. Comprehensive miRNome and in silico analyses identify the Wnt signaling pathway to be altered in the diabetic liver. Mol Biosyst 2011;7:3234-44.

41 Gurha P, Wang T, Larimore AH, et al. microRNA-22 promotes heart failure through coordinate suppression of PPAR/ERR-nuclear hormone receptor transcription. PLoS One 2013;8:e75882.

42 Hu Y, Liu H-X, Jena PK, et al. miR-22 inhibition reduces hepatic steatosis via FGF21 and FGFR1 induction. JHEP Rep 2020;2:100093. 
43 Davis JA, Saunders SJ, Mann M, et al. Combinatorial ensemble miRNA target prediction of co-regulation networks with nonprediction data. Nucleic Acids Res 2017;45:8745-57.

44 Lafontan M, Langin D. Lipolysis and lipid mobilization in human adipose tissue. Prog Lipid Res 2009;48:275-97.

45 Zechner R, Zimmermann R, Eichmann TO, et al. FAT SIGNALS-lipases and lipolysis in lipid metabolism and signaling. Cell Metab 2012;15:279-91.

46 Guilherme A, Virbasius JV, Puri V, et al. Adipocyte dysfunctions linking obesity to insulin resistance and type 2 diabetes. Nat Rev Mol Cell Biol 2008;9:367-77.
47 Ramsay RR, Popovic-Nikolic MR, Nikolic K, et al. A perspective on multi-target drug discovery and design for complex diseases. Clin Transl Med 2018;7:3.

48 Mechanick JI, Farkouh ME, Newman JD, et al. CardiometabolicBased chronic disease, adiposity and dysglycemia drivers: JACC state-of-the-art review. J Am Coll Cardiol 2020;75:525-38.

49 Hasib A. Multiagonist unimolecular peptides for obesity and type 2 diabetes: current advances and future directions. Clin Med Insights Endocrinol Diabetes 2020;13:1-8. 